PROCEEDINGS OF THE

AMERICAN MATHEMATICAL SOCIETY

Volume 126, Number 6, June 1998, Pages 1721-1724

S 0002-9939(98)04255-5

\title{
A UNIQUENESS THEOREM FOR HARMONIC FUNCTIONS
}

\author{
N. V. RAO
}

(Communicated by J. Marshall Ash)

Abstract. The main result of this note is the following theorem:

Theorem 1. Let $D=\left\{(x, t) ;|x|^{2}+t^{2} \leq r^{2}, t>0\right\}$ be a half ball in $R^{n+1}$ and $x \in R^{n}$. Assume that $u$ is $C^{1}$ in $\bar{D}$ and harmonic in $D$, and that for every positive integer $N$ there exists a constant $C_{N}$ such that

(1) $\quad|\nabla u(x, 0)| \leq C_{N}|x|^{N} \quad$ in a neighbourhood $V$ of the origin in $\partial D$;

$$
u(x, 0) \geq u(0,0) \text { in } \quad V .
$$

Then $u \equiv u(0,0)$.

First we prove it for $R^{2}$, and then we show by induction that it holds for all $n \geq 3$.

\section{INTRODUCTION}

Theorem 1 stated in the Abstract is somewhat analogous to theorem 1 of Baouendi and Rothschild [1], which is a uniqueness theorem for holomorphic functions of one complex variable; they applied it to obtain results on unique continuation for functions of several complex variables. For $n=2$, we show that our theorem is equivalent to theirs. In [2], Baouendi and Rothschild obtained results that are related to ours, but that neither imply nor are implied by ours. The main result of [2] is theorem 3 , where they assume that $u$ vanishes of infinite order at the origin in the normal direction and $u \geq 0$ in $V$, and deduce that $u$ vanishes on $V$ and also along the normal identically. In our case we assume that $\nabla u$ vanishes of infinite order at the origin restricted to $\partial D$, and deduce the same thing. We deduce uniqueness based on tangential behaviour, whereas they deduce uniqueness based on behaviour along the normal direction. But one might argue that $\nabla u$ includes the normal derivative. On the other hand it is not clear how, from the tangential decay of the normal derivative, one can deduce the decay of $u$ along the normal direction unless one assumes $u$ is $C^{\infty}$ on $\bar{D}$. Also in the infinitely smooth case one can easily see the equivalence of our results to those of [2].

For completeness we quote theorem 1 of [1]:

Theorem BR. Let $\Omega=\{z ;|z|<r, y>0\}$, a half disc in the complex plane $C$. Assume that $h$ is continuous in $\bar{\Omega}$ and holomorphic in $\Omega$, and that $h$ vanishes up to infinite order at the origin, i.e., for every positive integer $N$ there exists a constant

Received by the editors August 20, 1996 and, in revised form, November 20, 1996.

1991 Mathematics Subject Classification. Primary 31A05.

(C)1998 American Mathematical Society 
$C_{N}$ such that

$$
\begin{gathered}
\qquad|h(z)| \leq C_{N}\left|z^{N}\right|, z \in \bar{\Omega} ; \\
\operatorname{Re} h(x) \geq 0, x \in \partial \Omega \cap R \quad \text { in a neighbourhood of the origin. }
\end{gathered}
$$

Then $h \equiv 0$.

Condition (3) assumes that $h(z)$ vanishes up to infinite order from within $\Omega$, but one does not really need that. Theorem BR is equivalent to the following:

Theorem 2. In theorem BR, all else being equal, we replace (3) by the following: $h$ vanishes up to infinite order at 0 on $\partial \Omega$, i.e., for every positive integer $N$ there exists a constant $C_{N}$ such that

$$
|h(x)| \leq C_{N}|x|^{N}, \quad x \in \partial \Omega \cap R \quad \text { in a neighbourhood of } 0 .
$$

Then $h \equiv 0$.

Proof. We show that $\left(3^{\prime}\right)$ implies (3). We note that $\ln |h(z)|$ is subharmonic in $\Omega$; on $\partial \Omega$,

$$
\ln |h(z)| \leq N \ln |z|+\ln C_{N} \quad \text { in a neighbourhood of the origin, }
$$

and outside that neighbourhood

$$
\ln |h(z)|-N \ln |z|-\ln C_{N} \quad \text { is bounded above (say) by } C_{N}^{\prime}>0 .
$$

Therefore

$$
\ln |h(z)| \leq N \ln |z|+\ln C_{N}+C_{N}^{\prime} \text { on } \partial \Omega ;
$$

by the maximum principle,

$$
\ln |h(z)| \leq N \ln |z|+\ln C_{N}+C_{N}^{\prime} \text { on all of } \Omega,
$$

and so

$$
|h(z)| \leq C_{N} e^{C_{N}^{\prime}}|z|^{N} \quad \text { on } \Omega
$$

proving (3).

In order to deal with harmonic functions in $R^{2}$, we reformulate theorem 1 as follows:

Theorem 3. Suppose $u$ is harmonic in $\Omega$ and $C^{1}$ in $\bar{\Omega}$. Assume also that for every positive integer $N$, there exists a constant $C_{N}$ such that

$$
\begin{gathered}
|\nabla u(z)| \leq C_{N}|z|^{N} \text { for all } z \in \partial \Omega \text { in a neighbourhood of the origin; } \\
u(x) \geq u(0) \quad \text { for all } x \text { in a neighbourhood of the origin on } \partial \Omega .
\end{gathered}
$$

Then $u \equiv u(0)$.

Proof. Let $v(z)$ be the harmonic conjugate of $u$ such that $v(0)=0$. It is well-known that $v$ is continuous in $\bar{\Omega}$. Let $f(z)=u(z)+i v(z)-u(0)$. Clearly $f(z)$ is continuous on $\bar{\Omega}$, holomorphic in $\Omega$, and $\operatorname{Re} f \geq 0$ in a neighbourhood of the origin on $\partial \Omega$. We notice that

$$
\frac{\partial u}{\partial x}-i \frac{\partial u}{\partial y}=f^{\prime}(z), \int_{0}^{z} f^{\prime}(s) d s=f(z)
$$


Therefore, by (5), on $\partial \Omega$ in a neighbourhood of the origin we have

$$
|f(z)| \leq C_{N} \int_{0}^{|z|}|s|^{N}|d s|=\frac{C_{N}}{N+1}|z|^{N+1} .
$$

Now by theorem $2, f \equiv 0$ and so $u \equiv u(0)$.

We need the following lemma:

Lemma 4. Suppose $u$ is at least $C^{2}$ in a symmetric interval $[-r, r], u(x) \equiv u(-x)$, and

$$
v(x)=I(u)(x)=\int_{0}^{\frac{\pi}{2}} u(x \cos \theta) x \cos \theta d \theta .
$$

Then

$$
v^{\prime \prime}(x) \equiv I(L u)(x),
$$

where $L(u)$ is defined as follows:

$$
L(u)(x)=u^{\prime \prime}(x)+\frac{u^{\prime}(x)}{x} .
$$

This must be classical, but I do not know any good reference. Here is a short proof.

Proof. Differentiating under the integral sign twice, we obtain

$$
v^{\prime \prime}(x)=\int_{0}^{\frac{\pi}{2}} u^{\prime \prime}(x \cos \theta) x \cos ^{3} \theta+2 u^{\prime}(x \cos \theta) x \cos ^{2} \theta d \theta
$$

and further

$$
I(L(u))(x)=\int_{0}^{\frac{\pi}{2}} u^{\prime \prime}(x \cos \theta) x \cos \theta+u^{\prime}(x \cos \theta) d \theta .
$$

Hence

$$
\begin{aligned}
v^{\prime \prime}(x)-I(L(u))(x) & =\int_{0}^{\frac{\pi}{2}}\left(-u^{\prime \prime}(x \cos \theta) x \cos \theta \sin ^{2} \theta+u^{\prime}(x \cos \theta)\left(2 \cos ^{2} \theta-1\right)\right) d \theta \\
& =\int_{0}^{\frac{\pi}{2}} \frac{d}{d \theta}\left\{u^{\prime}(x \cos \theta) \sin \theta \cos \theta\right\} d \theta \\
& =\left.u^{\prime}(x \cos \theta) \sin \theta \cos \theta\right|_{0} ^{\frac{\pi}{2}} \\
& =0 .
\end{aligned}
$$

This proves the lemma.

Proof of theorem 1. For simplicity of notation we shall deal with $R^{3}$, but the method easily generalizes to $R^{n}$. Without loss of generality we may assume that $u(0,0,0)=$ 0 . Let

$$
u_{\theta}(x, y, t)=u(x \cos \theta-y \sin \theta, x \sin \theta+y \cos \theta, t) .
$$

Then $u_{\theta}$ is harmonic for any fixed $\theta$ in $D$, and is $C^{1}$ in $\bar{D}$. Let

$$
m(x, y, t)=\frac{1}{2 \pi} \int_{0}^{2 \pi} u_{\theta}(x, y, t) d \theta
$$


Then $m(x, y, t)$ is harmonic in $D$, and is $C^{1}$ in $\bar{D}$. But also $p(x, t)=m(x, 0, t)$ is $C^{1}$ in $\Omega$ [the half-disc in theorem $\mathrm{BR}$ ] and satisfies the differential equation

$$
\frac{\partial^{2} p}{\partial x^{2}}+\frac{1}{x} \frac{\partial p}{\partial x}+\frac{\partial^{2} p}{\partial t^{2}}=0
$$

Therefore by Lemma 4, the function

$$
v(x, t)=\int_{0}^{\frac{\pi}{2}} p(x \cos \theta, t) x \cos \theta d \theta
$$

is harmonic in $\Omega$ and $C^{1}$ in $\bar{\Omega}$. It is clear that

$$
|\nabla m| \leq \max _{0 \leq \theta \leq 2 \pi}\left|\nabla u_{\theta}\right|,|\nabla p(x, t)| \leq|\nabla m(x, 0, t)| .
$$

Therefore from (1) we have the estimate

(9) $\quad|\nabla p(x, 0)| \leq C_{N}|x|^{N}$ in a neighbourhood of the origin on the $x$-axis.

Also

$$
\begin{aligned}
& \frac{\partial v}{\partial x}=\int_{0}^{\frac{\pi}{2}} \frac{\partial p}{\partial x}(x \cos \theta, t) x \cos ^{2} \theta+p(x \cos \theta, t) \cos \theta d \theta \\
& \frac{\partial v}{\partial t}=\int_{0}^{\frac{\pi}{2}} \frac{\partial p}{\partial t}(x \cos \theta, t) x \cos \theta d \theta
\end{aligned}
$$

and further we notice that $|p(x, 0)-p(0,0)|=|p(x, 0)| \leq|x| \max _{|s| \leq|x|}|\nabla p(s, 0)| \leq$ $C_{N}|x|^{N+1}$. Combining (9) and (10), we get that in a neighbourhood of the origin on the $x$-axis,

$$
|\nabla v(x, 0)| \leq C_{N}|x|^{N+1}, v(x, 0) \geq 0 .
$$

Now applying theorem 3 to $v$, we have $v(x, t) \equiv 0$. Because $p$ is non-negative on the $x$-axis in a neighbourhood of the origin, this gives $p(x, 0)=0$ in a neighbourhood of the origin, and this in turn leads to the conclusion that $m(x, y, 0)=0$ and hence $u(x, y, 0)=0$ in a neighbourhood of the origin. But this would imply that $u$ and hence $\nabla u$ are real-analytic at the origin, and thus (1) implies $u \equiv 0$.

Remark 5 . We can reduce the general $R^{n}$ to the case of $R^{n-1}$ in the same way we reduced from $R^{3}$ to $R^{2}$.

Open problem. Suppose $u$ is continuously differentiable on the closed upper half of the unit disk $\Omega$ in $R^{2}$ and $\nabla u$ restricted to $\partial \Omega$ vanishes up to order $N$ at the origin. Then it is easy to show that it vanishes up to order $N$ at the origin from within $\Omega$ also. Is the same result true in higher dimensions?

\section{REFERENCES}

[1] M. S. Baouendi and L. P. Rothschild, Unique continuation and a Schwarz reflection principle for analytic sets, Comm. P. D. E. 18(11) (1993), pp. 1961-1970. MR 94i:32014

[2] M. S. Baouendi and L. P. Rothschild, A local Hopf lemma and unique continuation for harmonic functions, Int. Math. Res. Notices, 1993, no. 8, pp. 245-251. MR 94i:31008

Department of Mathematics, University of Toledo, Toledo, Ohio 43606

E-mail address: rnagise@uoft02.utoledo.edu 